\title{
A NOVEL VIEW-LEVEL TARGET BIT RATE DISTRIBUTION ESTIMATION TECHNIQUE FOR REAL-TIME MULTI-VIEW VIDEO PLUS DEPTH
}

\author{
Mario Cordina \\ Department of Communication and Computer \\ Engineering \\ University of Malta \\ Msida MSD2080, Malta \\ E-mail: mario.cordina@ieee.org
}

\begin{abstract}
This paper presents a novel view-level target bit rate distribution estimation technique for real-time Multi-view video plus depth using a statistical model that is based on the prediction mode distribution. Experiments using various standard test sequences show the efficacy of the technique, as the model manages to estimate online the view-level target bit rate distribution with an absolute mean estimation error of $2 \%$ and a standard deviation of $0.9 \%$. Moreover, this technique provides adaptation of the view-level bit rate distribution providing scene change handling capability.
\end{abstract}

Keywords- online estimation, multi-view video, view-level rate control, generalised linear models

\section{INTRODUCTION}

The latest advances in high speed networking together with improvements in video capturing devices, have given rise to a plethora of multimedia services. Moreover, customers are increasingly demanding a more realistic, immersive, multimedia experience. Multi-view Video (MVV) is appealing as it provides viewers with a 3D scene representation together with the ability to select and control the viewpoint. Such features allow MVV to be used in various applications such as tele-presence, tele-medicine, Free-View Video (FVV) and 3D TV $[1,2]$. With progress in multimedia capture and compression technologies together with the launch of the first commercially available full size auto-stereoscopic 3D display by Toshiba and Phillips [3], MVV technology is gaining ground in the entertainment and consumer arena. In addition, $3 \mathrm{D}$ video content is gradually permeating the consumer market through the proliferation of 3D Blu-ray disks, 3D broadcasts and the Internet [4].

There are several 3D video representations in literature, such as model-based representations [5] and point samplebased representations [6]. However, the Multi-view Video plus Depth (MVD) data format is being proposed as the main 3D representation format as it provides good rendering quality and flexible processing capabilities $[7,8]$. Such data format fulfils the 3D video system's requirements whilst supporting wide angle 3D displays and auto-stereoscopic displays [4]. Moreover, it allows the rendering of a continuum of output views with high image quality and low complexity [8] through the use of depth image-based rendering (DIBR) techniques. MVD needs the deployment of several video cameras to simultaneously capture a scene from different angles (views) and locations, leading to a

\author{
Carl J. Debono \\ Department of Communication and Computer \\ Engineering \\ University of Malta \\ Msida MSD2080, Malta \\ E-mail: c.debono@ieee.org
}

significant amount of data. For this reason, MVD is generally jointly compressed using the Multi-view Video Coding (MVC) standard by exploiting the redundancy in space, time and in between views $[9,10]$. Additionally, the depth map is often treated as the luminance component of color video, thereby allowing it to be compressed using either H264/AVC or H264/MVC. The resulting MVD stream is then transported over a bandwidth limited network and thus efficient rate control in the encoder is necessary to ensure that the 3D video coding satisfies the channel bandwidth and the decoder buffer constraints.

Rate control in MVC is still in its infancy and no rate control solution has yet been defined in the standard. Moreover, current work focuses on either extending the H264/AVC rate control solutions or the implementation of offline strategies which are not suitable for real-time video. MVD rate control is generally divided into three levels namely view-level, video/depth-level and frame/macro block-level. Correct estimation of the target bit rate at each level is essential for optimum operation. In this paper, we propose a view-level target bit rate distribution estimation technique for real-time MVD based on statistical analysis of the prediction modes used in the different view types.

The rest of the paper is organized as follows: Section 2 gives a summary of related work; Section 3 describes the proposed online view-level target bit rate distribution estimation technique together with an overview on statistical regression models; Section 4 gives a detailed account of the statistical model formulation and its performance while Section 5 provides some comments and conclusions.

\section{RELATED WORK}

Rate control in $2 \mathrm{D}$ video coding has garnered significant attention in the past decade and a number of algorithms have been proposed, including MPEG-2 TM5 [11], H.263 TMN8 [12] and the H264 rate control algorithm [13,14,15]. However, to the best knowledge of the authors, limited work exists on rate control for MVC and MVD video coding.

A 3D Multi-view rate control algorithm based on the human visual system was first proposed by Lim et al. [16], however this was not based on the new H264/MVC standard. Yan et al. proposed a Multi-view video rate control algorithm for H264/MVC [17] based on [13]. In their work, the authors first propose an improved quadratic rate-quantization (R-Q) model to cater for the additional prediction modes and frames in MVC. Then, using the 
fluid-flow traffic model, hypothetical reference decoder (HRD) and the improved R-Q model, they estimate the target bit rates for the group of group of pictures (GGOP), group of pictures (GOP) and frame levels.

Work on rate control for video plus depth representation was proposed in [18]. In this paper, the authors develop a rate control technique for $3 \mathrm{D}$ video based on Evolution Strategy (ES) for offline H264/AVC 3D video encoding using subjective quality assessment. The main drawback of this algorithm lies in the fact that it is not based on the new H264/MVC standard and is not suitable for real-time applications. The authors of [19] propose a similar approach using Lagrangian optimisation techniques.

Another aspect of rate control is the bit allocation problem. In [20], Morvan et al. studied the joint depth/texture bit allocation problem for multi-view video compression. In order to ensure efficient transmission of 3D video, the compression of both texture and depth images is required. They proposed a joint depth/texture bit allocation algorithm for the compression of MVD by combining the depth and texture rate-distortion (R-D) curves yielding a single R-D surface. A fast hierarchical optimisation algorithm employing an orthogonal search pattern exploits the smooth monotonic properties of the R-D surface, thereby allowing the optimisation of the joint bit-allocation problem in relation to the rendering quality. Although this joint model could be readily integrated into H264/MVC, it requires offline analysis to generate the R-D surface.

Another solution for MVD was developed by Liu et al. [21]. In their study, the authors propose a rate control technique for MVD based 3D video coding by using an image-stitching method to simultaneously encode video and depth. This is followed by a joint 3-level rate control algorithm made up of:

View level rate allocation: In MVC, different inter-view predictions can lead to a different R-D performance [21]. For instance, the I-view typically requires a higher bit rate than a P-view or a B-view at the same visual quality. From experiments conducted by the authors, they found that the average rate proportion among the three views (I-view, Bview and $\mathrm{P}$-view) for different quantisation parameter (QP) settings is approximately equal to $6: 4: 5$ for the Breakdancers sequence and 4.3:3.1:3.9 for the Ballet sequence [21]. The statistical rate allocation for a given sequence is pre-calculated, by offline encoding several frames from each view, and used to assign a different bit rate proportion to the different views depending upon the view type.

Video and depth allocation: Since the depth map is strictly used for the rendering of virtual views, the depth sequence can be significantly compressed to attain the required channel bandwidth constraint. In their work, the authors define the bit rate for the depth sequence $R_{d}$ as a fraction of the bit rate for the video sequence $R_{v}$. Using a linear R-Q model, the relationship between QP of the depth sequence, $Q_{d}$, and $\mathrm{QP}$ of the video sequence, $Q_{v}$, is given by:

$$
Q_{d}=Q_{v}+\left\lceil\log _{2}\left(\frac{K_{d}}{\alpha K_{v}+\left(\alpha C_{v}-C_{d}\right) * 2^{\frac{\left(Q_{v}-4\right)}{6}}}\right)\right\rceil
$$

where $K_{v}, K_{d}, C_{v}$, and $C_{d}$ are sequence dependent constants which are initialised after pre-encoding several frames from each view during the view-level rate allocation stage.

Frame-level rate control: The authors used a hierarchical rate allocation method together with coding complexity and buffer constraints to regulate the target bits for each frame [21]. Using the estimated target bits, the quantisation parameter is computed using the quadratic R-Q model [22]. Furthermore, according to the special characteristics of the Multi-view HRD, the buffer-related rate control is also considered to prevent the decoder buffer from overflow or underflow even when outputting multiple views.

Similar to other works, the main drawback of this technique is its dependency on offline processing. Moreover, the use of hierarchical $\mathrm{B}$ coding order is inherently restricting its use to non-real-time video sequences, as future frames are not known. Furthermore, it also precludes the handling of scene changes unless the complete video sequence is analysed apriori leading to a two-pass rate control technique.

\section{ONLINE VIEW-LEVEL TARGET Bit RATE DISTRIBUTION ESTIMATION}

Consider an advanced MVD-based 3D video system as proposed in [4]. Further assume that the MVC encoder is able to simultaneously encode multiple views. Through the use of DIBR techniques, views can be synthesised at the receiver side, thereby allowing us to encode and transmit a subset of the views. In this work, we are considering three views, namely the view 0 , view 1 and view 2 .

In general, video sequences are sequentially encoded using the H264/MVC hierarchical B coding structure. Although this yields a better compression ratio, it comes at the expense of a higher coding delay as by the time the reference frames are available, the current frame being decoded should have been already displayed. Real-time applications such as video conferencing are very sensitive to delay and industry standards limit the end-to- end delay to a maximum of $300 \mathrm{~ms}$ making such prediction structure unsuitable to real-time communications. By default, the current Joint Multi-view Video Coding (JMVC) software model uses the hierarchical B coding structure however, as from JMVC ver. 2.3, the model supports a GOP size of 1 which allows the use of an I-P-P-P structure in the time domain. When using this GOP setting, the coding structure needs to be defined in the configuration file, whereby for each view, the reference frames that may be used must be 
specified. By setting the appropriate references, a real-time prediction structure, utilising inter-view B frames as shown in Fig. 1, can be implemented without additional time delay as these can be catered for by parallel hardware.

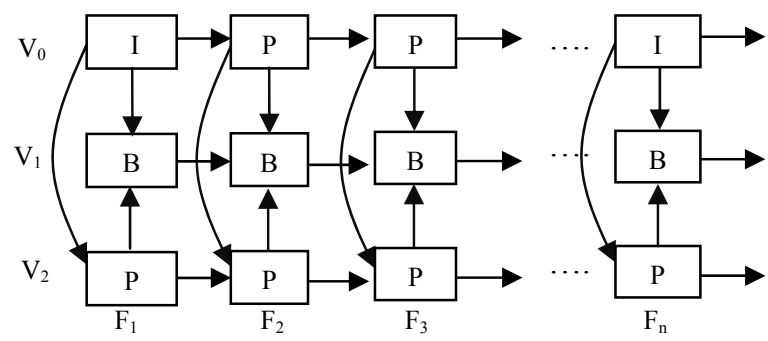

Figure 1. Real-time prediction structure.

A key aspect in such a rate control framework is the estimation of the allocated bit rate in proportion to the different views, depending on the view type. This viewlevel target bit rate allocation defines the target bit rate bounds of the video/depth and the frame layer rate control algorithm. Ideally, this estimation is carried out periodically and in real-time to adapt to the changing video sequence. Moreover, it cannot make use of the controlled bit rate values at the output of the rate controller as this directly depends on the correct estimate at the input. However, as we have seen in section II, the view-layer target bit rate distribution is usually estimated using offline processing techniques with no adaptation. By exploiting the distribution of the prediction modes in the different views, which apart from other factors depends on the video sequence characteristics and prediction structure, we can infer the bit rate allocation for the different views.

To apply adaptation, we propose the use of a statistical regression model, which has been trained offline, to estimate online the view-level target bit rate distribution, as shown in Figure 2. This estimation is carried out periodically (say at every anchor period) in order to provide scene change handling. The distribution of the prediction modes has a direct impact on the bit rate. Therefore, by observing the statistical distribution of the Macroblock (MB) prediction modes, view type (I, P or B) and the viewlevel bit rate for various video sequences and QPs, a statistical regression model can be formulated.

In JMVM, the supported MB prediction modes include SKIP, DIRECT, Inter16x16, Inter16x8, Inter8X16, Inter8x8, Inter8x8Frext, Intra16x16, Intra8x8 and Intra4x4. The prediction mode with the lowest R-D cost [3], given by (2), is chosen to encode the MB.

$$
J\left(S_{k}, I_{k} \mid \lambda\right)=\operatorname{SSD}\left(S_{k}, I_{k}\right)+\lambda R\left(S_{k}, I_{k}\right)
$$

where $S_{k}$ and $I_{k}$ denote the $k^{\text {th }} \mathrm{MB}$ and the corresponding MB mode respectively, $\lambda$ is the Lagrange multiplier for mode decision, $\operatorname{SSD}\left(S_{k}, I_{k}\right)$ is the sum of squared difference between the reconstructed $\mathrm{MB}$ and the original $\mathrm{MB}$, and $R\left(S_{k}, I_{k}\right)$ is the rate after entropy coding.

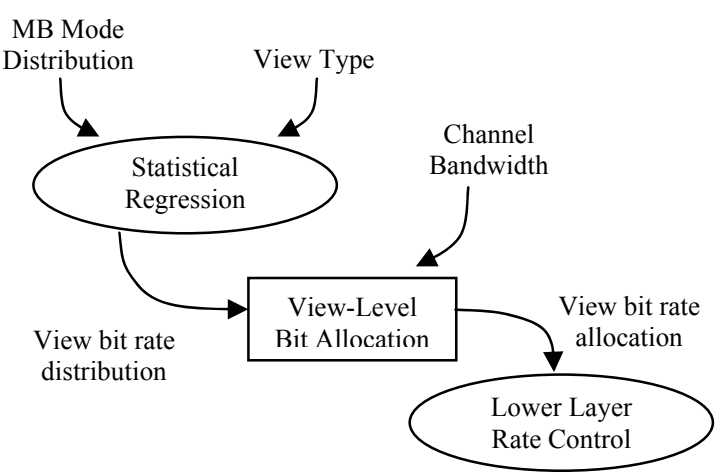

Figure 2. Overview of bit rate distribution estimation.

There are several regression models in literature, however linear regression models are the most commonly used due to their well-known statistical properties and are typically composed of a single dependent variable (viewlevel bit rate) and several predictors (QP, view type and prediction modes). A well known general linear model that accommodates predictors which are a combination of factors and covariates, as in our case, is the Analysis of Covariance (ANCOVA). For $n$ observed cases, the ANCOVA model that expresses the dependent variable $y_{n}$ as a function of $p$ predictors $\beta_{p}$ is given by [23]:

$$
\begin{gathered}
\left(\begin{array}{c}
y_{1} \\
\vdots \\
y_{n}
\end{array}\right)=\left(\begin{array}{ccc}
x_{11} & \cdots & x_{1 p} \\
\vdots & \ddots & \vdots \\
x_{n 1} & \cdots & x_{n p}
\end{array}\right)\left(\begin{array}{c}
\beta_{1} \\
\vdots \\
\beta_{p}
\end{array}\right)+\left(\begin{array}{c}
\varepsilon_{1} \\
\vdots \\
\varepsilon_{n}
\end{array}\right) \\
\mathbf{y}=\mathbf{x} \beta+\varepsilon
\end{gathered}
$$

where $\beta$ is a $p$-vector of unknown regression parameters estimated using least squares estimation, $\varepsilon$ is an $n$-vector of unknown random error terms, $\mathbf{y}$ is an $n$-vector of responses which apart from having a normal distribution, it is also linearly related and highly correlated with each quantitative predictor, and $\mathbf{x}$ is an $n \times p$ matrix whose elements are a mixture of real and dummy ( 0 or 1 indicators) values and are uncorrelated with each other.

Given that the errors have a normal distribution, the least square estimate, which is equivalent to the maximum likelihood estimator $b$, is given by:

$$
b=\left(\mathbf{x}^{\prime} \mathbf{x}\right)^{-1} \mathbf{x}^{\prime} \mathbf{y}
$$

Although regression models, such as the ANCOVA model, have been used extensively by researchers for prediction, inference and modelling, these models rely heavily on the underlying assumptions. In particular, regression models assume that the dependent variable has a normal distribution and that the predictors are independent. However, these assumptions are not always valid and other modelling techniques have to be explored. One of the most significant contributions in statistical modelling is the 
concept of generalised linear models (GLM) [24]. These models relate the dependent variable to the linear predictors through any invertible link function and accommodate any error distribution present in the exponential family. Although GLM can be viewed as a generalisation of the general linear model, they still rely on the assumption that the responses are independent. GLM are characterised by three components:

1) For $n$ observed cases, the dependent variable $y_{n}$ is assumed to be independent and follows a distribution that is a member of the exponential family.

2) The predictors influence the distribution of the dependent variable $y_{n}$ through a single linear function known as the linear predictor and given by:

$$
\eta_{i}=\beta_{1} x_{1 i}+\beta_{2} x_{2 i}+\ldots .+\beta_{p} x_{p i}=\mathbf{x} \beta
$$

3) The mean $\mu=E(\mathbf{y})$ is related to the linear predictor through an invertible link function $g(\mu)$.

$$
g\left(\mu_{i}\right)=\eta_{i}=\mathbf{x} \beta
$$

Thus, the GLM is given by $\mathrm{E}(\mathbf{y})=\mu=\mathrm{g}^{-1}(\mathbf{x} \beta)$ where $\mathbf{y}$ is an $n$-vector of responses, $\beta$ is a $p$-vector of unknown regression parameters estimated using maximum likelihood techniques, and $\mathbf{x}$ is an $n \times p$ matrix whose elements are a mixture of real and dummy ( 0 or 1 indicators) values.

\section{Statistical Model Formulation}

The proposed online view-level target bit rate distribution estimation technique was implemented in two parts: First the statistical model was formulated, and then the statistical model integrated in JMVC ver. 8.3.1. The latter also included the verification of its effectiveness in estimating the view-level target bit rate distribution. Prior to the statistical model formulation, the JMVC model was modified to extract information such as the prediction mode distribution, view type, and average bit rate for various QP settings, parse it and import it into IBM SPSS ${ }^{\odot}$ statistical analysis package for analysis. The encoding parameters together with the test sequences used and their respective characteristics are shown in Table I and II respectively.

TABLE I. ENCODING PARAMETERS

\begin{tabular}{|c|c|}
\hline GOP Size & 1 \\
\hline Intra Period & 12 \\
\hline QP & 26 to 50 \\
\hline Prediction Structure & Real-time \\
\hline Encoded Frames & 60 \\
\hline
\end{tabular}

Before selecting a statistical regression model, the dependent variable was checked for normality using the One-Sample Kolmogorov-Smirnov test at the 0.05 level of significance. The result of this test, indicates that the data is not normally distributed, as the $p$-value is lower than 0.05 , thereby eliminating the use of a general linear model, such as the ANCOVA model. This was further confirmed after plotting the histogram of the dependent variable, as shown in Fig. 3.

TABLE II. TEST SEQUENCE PARAMETERS AND CHARACTERISTICS ( ${ }^{\vee}$ INDICATES THE SEQUENCE IS USED FOR VERIFICATION ONLY)

\begin{tabular}{|c|c|c|c|}
\hline Sequence & Image Size & $\begin{array}{c}\text { Frame } \\
\text { Rate }\end{array}$ & Features \\
\hline Kendo $^{\mathrm{V}}$ & $1024 \times 768$ & 30 & $\begin{array}{c}\text { Complex object motion; } \\
\text { Moving camera }\end{array}$ \\
\hline $\begin{array}{c}\text { Break } \\
\text { dancers }\end{array}$ & $1024 \times 768$ & 15 & $\begin{array}{c}\text { Slow and very fast motion; } \\
\text { No camera motion }\end{array}$ \\
\hline Balloons & $1024 \times 768$ & 30 & $\begin{array}{c}\text { Complex object motion; } \\
\text { Moving camera }\end{array}$ \\
\hline $\begin{array}{c}\text { Champ. } \\
\text { Tower }\end{array}$ & $1280 \times 960$ & 30 & $\begin{array}{c}\text { Slow but complex object } \\
\text { motion; No camera motion }\end{array}$ \\
\hline $\begin{array}{c}\text { Book } \\
\text { Arrival }\end{array}$ & $1024 \times 768$ & 15 & $\begin{array}{c}\text { Moderate object motion; } \\
\text { No camera motion }\end{array}$ \\
\hline
\end{tabular}

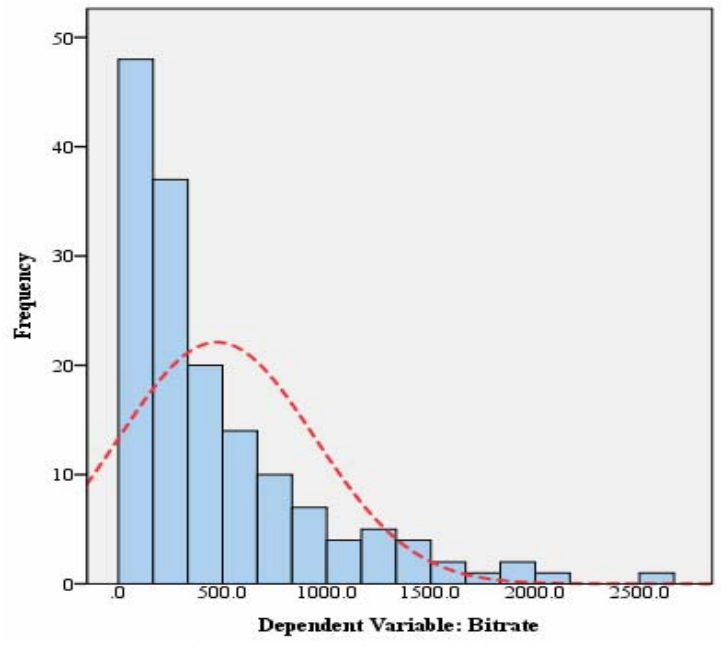

Figure 3. Dependent Variable Histogram.

This shows that the dependent variable follows a distribution which is a member of the exponential family. Therefore, a generalised linear model using the identity link function was chosen to fit the data. The initial GLM parameter estimates together with the Wald-Chi square and confidence levels are shown in Table III. As shown in this table, the Mode 16x16 and Mode16x8 have a $p$-value which is higher than the 0.05 level of significance. This implies that their contribution in explaining the variation in the bit rate was not found to be significant and thus these two predictors need to be removed from the model fitting system. Excluding these two predictors and re-calculating the parameter estimates we obtain the parsimonious GLM model shown in Table IV.

Thus, the parsimonious GLM model is composed of 10 significant predictors and is given by (8).

$B=1945.5-179.2 V_{1}+161.9 V_{2}+1903.7$ Direct $B-$

13472.9Mode8x16+2021.7Intra4 $44+61861.8$ Mode8 $x 8-$

3073.5Intra16x16-1349.4Skip-9.9QP 
where $B$ is the estimated view-level bit rate; DirectB, Mode8x16, Intra4x4, Mode8x8, Intra16x16 and Skip are ratios based on the $\mathrm{MB}$ prediction mode distribution over a number of frames or anchor period, QP is the quantisation parameter whereas $V_{1}$ and $V_{2}$ are flags used to indicate view type. $V_{1}$ and $V_{2}$ are set to 0 and 1 for an I-view, 1 and 0 for a B-view, 1 and 1 for a P-View respectively.

TABLE III. INITIAL GLM MODEL

\begin{tabular}{|c|c|c|c|}
\hline Parameter & Coefficient & Wald Chi-Square value & $\boldsymbol{p}$-value \\
\hline Constant Term & -700.3 & 16.137 & 0.000 \\
\hline V1 & -179.71 & 12.975 & 0.000 \\
\hline V2 & 150.31 & 19.163 & 0.000 \\
\hline DirectB & 3192 & 35.903 & 0.000 \\
\hline Mode16x16 & 851.72 & 1.888 & 0.169 \\
\hline Mode16x8 & 14195.62 & 3.304 & 0.069 \\
\hline Mode8x16 & -21459.65 & 9.288 & 0.002 \\
\hline Intra4x4 & 2878.49 & 7.729 & 0.005 \\
\hline Mode8x8 & 56273.25 & 33.154 & 0.000 \\
\hline Intra16x16 & -1725.3 & 17.253 & 0.000 \\
\hline QP & -11.38 & 14.48 & 0.000 \\
\hline
\end{tabular}

TABLE IV. PARSIMONOUS GLM MODEL

\begin{tabular}{|c|c|c|c|}
\hline Parameter & Coefficient & Wald Chi-Square value & $\boldsymbol{p}$-value \\
\hline Constant Term & 1945.51 & 16.06 & 0.000 \\
\hline V1 & -179.17 & 12.63 & 0.000 \\
\hline V2 & 161.99 & 23.22 & 0.000 \\
\hline Direct B & 1903.65 & 4.93 & 0.026 \\
\hline Mode 8x16 & -13472.9 & 7.43 & 0.006 \\
\hline Intra 4x4 & 2021.7 & 4.13 & 0.042 \\
\hline Mode8x8 & 61861.78 & 48.24 & 0.000 \\
\hline Intra 16x16 & -3073.47 & 22.49 & 0.000 \\
\hline Skip & -1349.43 & 5.85 & 0.016 \\
\hline QP & -9.927 & 11.95 & 0.001 \\
\hline
\end{tabular}

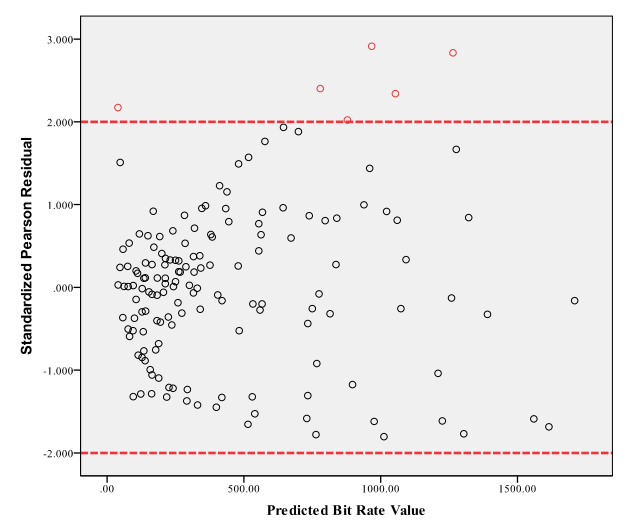

Figure 4. Standardised Pearson Residual.

The Standardised Pearson Residual [25], shown in Fig. 4 , is plotted against the predicted bit rate value to check for the efficacy of the fitted GLM model. The points in the Standardised Pearson Residual plot are largely spread across a horizontal band indicating that the residuals have a constant variance (homoscedasticity). An element of heteroscedasticity in the model is present as the variance increases slightly with the predicted value. However, since the majority of the points lie between the standard \pm 2 limit, the model is adequate for such data fit with a very small number of outliers (shown in red).

The GLM model was integrated within the JMVC framework and the estimated view-level bit rate distribution was calculated online from the estimation of the view-level bit rate of each view. This was done for all the test sequences and the plots of the estimated and actual viewlevel bit rate distribution during Model Formulation and Verification are shown in Fig. 5 and 6 respectively.

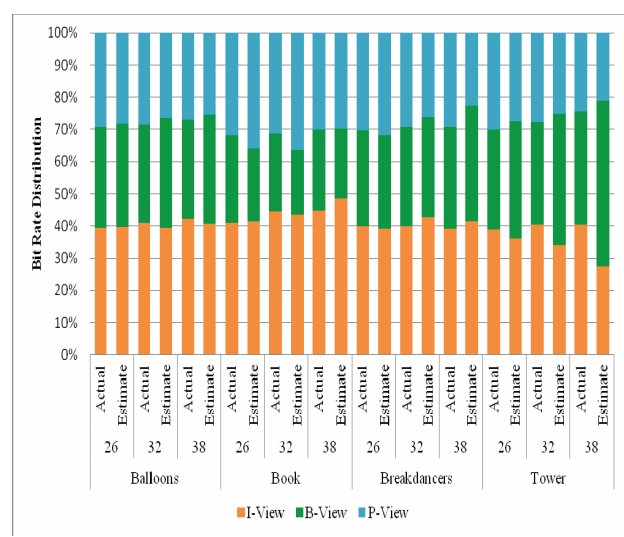

Figure 5. Bit Rate Distribution - Model Formulation.

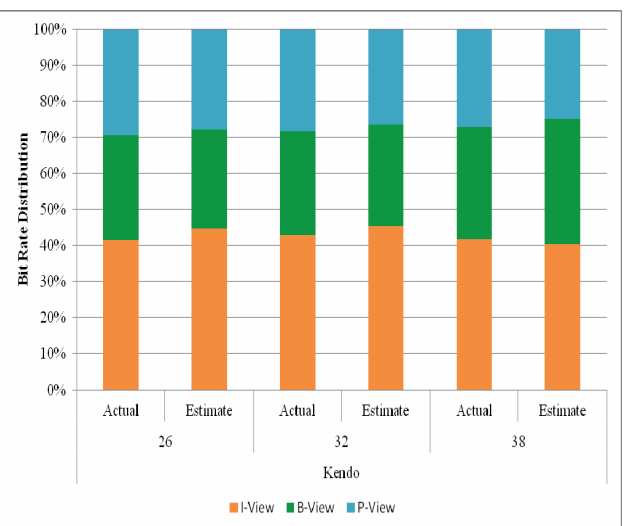

Figure 6. Bit Rate Distribution - Model Verification.

The mean and standard deviation of the estimation error for the test sequences used in the model formulation and verification are shown in Table $\mathrm{V}$. The formulated statistical regression model was able to estimate the view-level bit rate distribution with an overall estimation error of $3.48 \%$ and a standard deviation of $3.42 \%$ for the test sequences used during the model formulation. With reference to Fig. 6, it is noted that the model manages to accurately estimate the bit rate for sequences which were not used in model 
formulation with an estimation error of around $2 \%$ and standard deviation of $0.9 \%$. Although this is an acceptable error for target view-level bit rate setting, this performance can be further improved by including other test sequences during model formulation.

TABLE V. MODEL ESTIMATION ERROR

\begin{tabular}{|c|c|c|c|c|c|c|c|c|}
\hline \multirow{3}{*}{ Test Sequence } & \multicolumn{4}{|c|}{$\begin{array}{c}\text { \% Absolute Mean } \\
\text { Estimation error }\end{array}$} & \multicolumn{3}{c|}{$\begin{array}{c}\text { \% Standard Deviation of } \\
\text { the Estimation error }\end{array}$} \\
\cline { 2 - 9 } & $\begin{array}{c}\text { I- } \\
\text { View }\end{array}$ & $\begin{array}{c}\text { B- } \\
\text { View }\end{array}$ & $\begin{array}{c}\text { P- } \\
\text { View }\end{array}$ & $\begin{array}{c}\text { Over } \\
\text { all }\end{array}$ & $\begin{array}{c}\text { I- } \\
\text { View }\end{array}$ & $\begin{array}{c}B- \\
\text { View }\end{array}$ & $\begin{array}{c}P- \\
\text { View }\end{array}$ & $\begin{array}{c}\text { Over } \\
\text { all }\end{array}$ \\
\hline Model Formulation & 3.06 & 4.64 & 2.72 & 3.48 & 3.56 & 4.34 & 1.88 & 3.42 \\
\hline Model Verification & 2.31 & 1.92 & 1.96 & 2.02 & 1.0 & 1.4 & 0.35 & 0.9 \\
\hline
\end{tabular}

\section{CONCLUSION}

In this paper, we have presented a view-level target bit rate distribution estimation technique that can be applied to real time MVD systems based on a statistical regression model. Simulation results on sequences exhibiting a variety of video characteristics have shown that the technique is able to estimate the view-level target bit rate distribution in real-time with an absolute mean estimation error of $2 \%$ for the test sequences not used during model formulation. The model integrated in the JMVC framework allows view-level target bit rate setting for the underlying rate control algorithm whilst allowing scene change handling capability.

\section{ACKNOWLEDGMENT}

The authors would like to thank the Interactive Media Group of Microsoft Research for providing the Breakdancers test sequences, Nagoya University for providing Balloons, Kendo \& Champagne Tower test sequences and Fraunhofer-HHI for providing Book Arrival test sequence.

\section{REFERENCES}

[1] A. Smolic, P. Kauff, "Interactive 3-D Video representation and coding technologies," in Proc. of the IEEE, vol.93, no.1, pp. 98-110, Jan 2005.

[2] Y.S. Ho, K.J. Oh, "Overview of multiview video coding," in Proc. of International Workshop on Systems, Signals and Image Processing, pp. 5-12, Jun 2007.

[3] International Consumer Electronics Show 2011. Internet: http://www.cesweb.org

[4] A. Vetro, S. Yea and A. Smolic, "Towards a 3D video format for auto-stereoscopic displays," in Proc. Of SPIE: Appl. Digital Image Process. XXXI, Vol. 7073, Sep. 2008.

[5] C. Theobalt, G. Ziegler, M. Magnor, and H.P. Seidel, "Model-based free-viewpoint video acquisition, rendering and encoding," in Proc. Picture Coding Symp., San Francisco, pp. 1-6, Dec. 2004.
[6] S. Wurmlin, E. Lamboray, and M. Gross, "3D video fragments: Dynamic point samples for real-time free-viewpoint video," Computers \& Graphics, vol. 28, no. 1, pp. 3-14, Feb. 2004.

[7] Y.K. Park, K. Jung, Y. Oh, S. Lee, J.K. Kim, H. Lee, K. Yun, N. Hur and J. Kim, "Depth-image-based rendering for 3DTV service over T-DMB," Signal Process.: Image Commun., vol. 24, no. 1-2, pp. 122-136, Jan. 2009.

[8] P. Kauff, N. Atzpadin, C. Fehn, K. Müller, O. Schreer, A. Smolic, and R. Tanger, "Depth map creation and image-based rendering for Advanced 3DTV services providing interoperability and scalability," Signal Process.: Image Commun., vol. 22, no. 2, pp. 217-234, Feb. 2007.

[9] P. Merkle, A. Smolic, K. Müller, and T. Wiegand, "Efficient prediction structures for multi-view video coding," IEEE Trans. Circuits \& Syst. For Video Technol., vol. 17, no. 11, pp. 1461-1473, Nov. 2007.

[10] L.Q. Shen, Z. Liu, S.X. Liu, Z.Y. Zhang, and P. An, "Selective disparity estimation and variable size motion estimation based on motion homogeneity for multi-view coding," IEEE Trans. on Broadcast., vol. 55, no. 4, pp. 761-766, Dec. 2009.

[11] "MPEG 2 Test Model 5", Rev. 2 ISO/IEC JTC1/SC29WG11, Apr. 1993.

[12] J. Ribas-Corbera and S. Lei, "Rate control for low-delay video communications", ITU Study Group 16, VCEG, Portland, Doc. Q15-A-20, 1997.

[13] Z.G. Li, F. Pan, K.P. Li, G. Feng, X. Lin, and S. Rahardja, "Adaptive basic unit layer rate control for JVT", Pataya II, Thailand, Doc. JVT-G012e1, 2003.

[14] S. Ma, F. Wu, and Z. Li, "Proposed draft of adaptive rate control", Geneva, Switzerland, Doc. JVT-H017r3, 2003.

[15] Z. Min, T. Ikenaga, and S. Goto, "A Novel Rate Control Algorithm for H.264/AVC", 23rd International Technical Conference on Circuits/Systems, Computers and Communications, 2008.

[16] J.E. Li, J. Ki, K.N. Ngan, and K. Sohn, "Advanced rate control technologies for 3D-HDTV", IEEE Trans. Consumer Electronics, vol. 49, no.4, pp. 1498-1507, Nov. 2003.

[17] T. Yan, P. An, L. Shen, Z. Li, H. Wang, Z. Zhang, "Frame-layer rate control algorithm for multi-view video coding", GEC Summit 2009, pp. 1025-1028, 2009.

[18] S.L.P. Yasakethu, W.A.C. Fernando, and A. Kondoz, "Rate controlling in offline 3D video coding using evolution strategy", IEEE Trans. Consumer Electronics, vol. 55, no. 1, pp. 150-157, Feb. 2009.

[19] B. Kamolrat, W.A.C. Fernando, and M. Mrak, "Rate controlling for colour and depth 3D video coding", in Proc. SPIE: Appl. Digital Image Process. XXXI, San Diego, CA, Aug. 2008.

[20] Y. Morvan, D. Farin and, P. De With, "Joint Depth/Texture bitallocation for multi-view video compression", Picture coding Symposium, Nov. 2007

[21] Y. Liu, Q. Huang, S. Ma, D. Zhao, W. Gao, S. Ci, and H. Tang, "A Novel Rate Control Technique for Multiview Video Plus Depth Based 3D Video Coding", IEEE Transactions on Broadcasting, pp. 562-571, Jun. 2011.

[22] H.J. Lee, T. Chiang, and Y.Q. Zhang, "Scalable rate control for MPEG-4 video", IEEE Trans. Circuits Syst. Video Technol., vol. 10, no. 6, pp. 878-894, Sep. 2000.

[23] K.V. Mardia, J.T. Kent and J.M. Bibby, Multivariate Analysis (Probability and Mathematical Statistics), Academic Press, Edition 1, Feb. 1980.

[24] P. McCullagh, J.A. Nelder, Generalized Linear Models, Chapman and Hall, $2^{\text {nd }}$ Edition, Aug. 1989.

[25] M. Norusis, IBM SPSS Statistics 19 Advanced Statistical Procedures Companion, Pearson, Feb. 2011. 